hep-th/0403082

March, 2004

\title{
Relaxed super self-duality and effective action
}

\author{
S. M. Kuzenko and I. N. McArthur \\ School of Physics, The University of Western Australia \\ Crawley, W.A. 6009, Australia \\ kuzenko@cyllene.uwa.edu.au, mcarthur@physics.uwa.edu.au
}

\begin{abstract}
A closed-form expression is obtained for a holomorphic sector of the two-loop Euler-Heisenberg type effective action for $\mathcal{N}=2$ supersymmetric QED derived in hep-th/0308136. In the framework of the background-field method, this sector is singled out by computing the effective action for a background $\mathcal{N}=2$ vector multiplet satisfying a relaxed super self-duality condition. The approach advocated in this letter can be applied, in particular, to the study of the $\mathcal{N}=4$ super YangMills theory on its Coulomb branch.
\end{abstract}


The two-loop extension of the (one-loop) QED Euler-Heisenberg action [1, 2, 3] was derived by Ritus [4] almost thirty years ago. Unlike the original (one-loop) Euler-Heisenberg action, its two-loop extension [4] involves a rather complicated double proper-time integral. It has recently been demonstrated [5] that for a self-dual background the two-loop QED effective action [4] takes a remarkably simple form, and actually becomes very similar to the one-loop action $[1,2,3]$ in the same background.

The Euler-Heisenberg Lagrangian corresponds to an approximation of slowly varying fields, and is a function of the field strength $F_{a b}$ only,

$$
L_{\mathrm{EH}}=L\left(F_{+}^{2}, F_{-}^{2}\right) .
$$

Here $F_{+}$and $F_{-}$are the (anti) self-dual components of the field strength $F$,

$$
\begin{array}{rlrl}
F_{ \pm} & =\frac{1}{2}(F \mp \mathrm{i} \tilde{F}), & \widetilde{F_{ \pm}} & = \pm \mathrm{i} F_{ \pm}, \\
F_{ \pm}^{2}=-\frac{1}{2}(\mathcal{F} \mp \mathrm{i} \mathcal{G}) \mathbf{1}_{4}, & \mathcal{F} & =\frac{1}{4} F^{a b} F_{a b}, \quad \mathcal{G}=\frac{1}{4} F^{a b} \tilde{F}_{a b},
\end{array}
$$

with $\tilde{F}$ the Hodge-dual of $F$. The general structure of $L_{\mathrm{EH}}$ is as follows:

$$
L\left(F_{+}^{2}, F_{-}^{2}\right)=\Lambda\left(F_{+}^{2}\right)+\bar{\Lambda}\left(F_{-}^{2}\right)+F_{+}^{2} F_{-}^{2} \Omega\left(F_{+}^{2}, F_{-}^{2}\right) .
$$

Choosing a self-dual background, $F_{-}=0$, one can keep track of the holomorphic function $\Lambda$, but all the information about the function $\Omega$ is lost.

In supersymmetric theories, there are no quantum corrections to $\Lambda$ and $\bar{\Lambda}$ beyond second order in the field strength, $\Lambda \propto F_{+}^{2}$, since no appropriate superfield invariant exists. In other words, the effective Lagrangian is essentially trivial in the case of selfdual fields (see $[6,7,8,9]$ for an incomlete list of references), and therefore the results of [5] cannot be applied directly. Fortunately, we can still profit, although rather indirectly, from the approach advocated in [5]. The point is that the function $\Omega$ in (3) has the following general form

$$
\Omega\left(F_{+}^{2}, F_{-}^{2}\right)=\omega\left(F_{+}^{2}\right)+\bar{\omega}\left(F_{-}^{2}\right)+F_{+}^{2} F_{-}^{2} \Upsilon\left(F_{+}^{2}, F_{-}^{2}\right) .
$$

Its holomorphic part, $\omega\left(F_{+}^{2}\right)$, can be restored by computing the effective action for background vector supermultiplets satisfying a relaxed self-duality condition.

In superfield notation, a relaxed super self-duality condition can be defined by

$$
W_{\alpha} \neq 0, \quad D_{\alpha} W_{\beta}=0, \quad \bar{D}_{(\dot{\alpha}} \bar{W}_{\dot{\beta})} \neq 0
$$


in the case of $\mathcal{N}=1$ supersymmetry, or

$$
D_{\alpha}^{i} W \neq 0, \quad D_{\alpha}^{i} D_{\beta}^{j} W=0, \quad \bar{D}_{(\dot{\alpha}}^{i} \bar{D}_{\dot{\beta}) i} \bar{W} \neq 0
$$

in the case of $\mathcal{N}=2$ supersymmetry. Here $W_{\alpha}$ and $W$ are the chiral superfield strengths describing the $\mathcal{N}=1$ and $\mathcal{N}=2$ Abelian vector multiplets, respectively. Ordinary (Euclidean) super self-duality [10] corresponds to setting $W=0$ while keeping $\bar{W}$ nonvanishing (see also $[9,11]$ ). From the point of view of $\mathcal{N}=1$ supersymmetry, the $\mathcal{N}=2$ vector multiplet strength $W$ consists of two $\mathcal{N}=1$ superfields: (i) a chiral scalar $\Phi$; and (ii) the $\mathcal{N}=1$ vector multiplet strength $W_{\alpha}$. The conditions on $W_{\alpha}$ which follow from (6) coincide with (5).

The condition of relaxed super self-duality has a simple meaning at the component level. In the case of an off-shell $\mathcal{N}=2$ vector multiplet, its chiral strength $W$ is known to contain the following component fields (with $U|\equiv U(x, \theta)|_{\theta=0}$ ): (i) a complex scalar $\varphi=W \mid$; (ii) two left-handed spinors $\psi_{\alpha}^{i}=D_{\alpha}^{i} W \mid$; (iii) a symmetric bi-spinor $F_{\alpha \beta}=$ $D_{(\alpha}^{i} D_{\beta) i} W \mid$ which is in one-to-one correspondence with $F_{-}$; (iv) an auxiliary iso-triplet $X^{i j}=D^{\alpha(i} D_{\alpha}^{j)} W \mid$. The relaxed super self-duality requires $F_{\alpha \beta}=X^{i j}=0$ and allows for non-vanishing $\varphi$ and $\psi_{\alpha}^{i}$. This is clearly a relaxation of the ordinary super self-duality requirements $\varphi=\psi_{\alpha}^{i}=F_{\alpha \beta}=X^{i j}=0$.

In Minkowski space-time, the conditions (5) and (6) are purely formal, as they are obviously inconsistent with the structure of a single real vector multiplet. Nevertheless, their use is completely legitimate if we are only interested in computing some special, holomorphic-like sector of the effective action. To be more specific, let us consider $\mathcal{N}=2$ supersymmetric QED (SQED).

The action of $\mathcal{N}=2$ SQED written in terms of $\mathcal{N}=1$ superfields is

$$
\begin{aligned}
S_{\mathrm{SQED}}= & \frac{1}{e^{2}} \int \mathrm{d}^{8} z \bar{\Phi} \Phi+\frac{1}{e^{2}} \int \mathrm{d}^{6} z W^{\alpha} W_{\alpha} \\
& +\int \mathrm{d}^{8} z\left(\bar{Q} \mathrm{e}^{V} Q+\overline{\bar{Q}} \mathrm{e}^{-V} \tilde{Q}\right)+\left(\mathrm{i} \int \mathrm{d}^{6} z \tilde{Q} \Phi Q+\text { c.c. }\right)
\end{aligned}
$$

where $W_{\alpha}=-\frac{1}{8} \bar{D}^{2} D_{\alpha} V$. The dynamical variables $\Phi$ and $V$ describe an $\mathcal{N}=2$ Abelian vector multiplet, while the superfields $Q$ and $\tilde{Q}$ constitute a massless Fayet-Sohnius hypermultiplet. The case of a massive hypermultiplet is obtained from (7) by the shift $\Phi \rightarrow \Phi+m$, with $m$ a complex parameter. ${ }^{1}$

\footnotetext{
${ }^{1}$ The action of $\mathcal{N}=1$ SQED is obtained from (7) by discarding $\Phi$ as a dynamical variable, and instead 'freezing' $\Phi$ to a constant value $m$.
} 
We are interested in a low-energy effective action $\Gamma[W, \Phi]$ which describes the dynamics of the $\mathcal{N}=2$ massless vector multiplet and which is generated by integrating out the massive charged hypermultiplet. More precisely, we concentrate on a slowly varying part of $\Gamma[W, \Phi]$ that, at the component level, comprises contributions with (the supersymmetrization of) all possible powers of the gauge field strength without derivatives. Its generic form is [12]

$$
\Gamma[W, \Phi]=\left(\alpha \int \mathrm{d}^{6} z W^{2} \ln \frac{\Phi}{\mu}+\text { c.c. }\right)+\int \mathrm{d}^{8} z \frac{\bar{W}^{2} W^{2}}{\bar{\Phi}^{2} \Phi^{2}} \Omega\left(\Psi^{2}, \bar{\Psi}^{2}\right),
$$

where

$$
\bar{\Psi}^{2}=\frac{1}{4} D^{2}\left(\frac{W^{2}}{\bar{\Phi}^{2} \Phi^{2}}\right), \quad \Psi^{2}=\frac{1}{4} \bar{D}^{2}\left(\frac{\bar{W}^{2}}{\bar{\Phi}^{2} \Phi^{2}}\right),
$$

$\mu$ is the renormalization scale and $\Omega$ some real analytic function. The first term on the right hand side of (8) is known to be one-loop exact in perturbation theory (see, e.g., [13]), while the second term receives quantum corrections at all loops $[12,15]$.

Up to a scale transformation, the function $\Omega$ in (8) is the same as the one in (3). To compute its holomorphic part, $\Omega\left(\Psi^{2}, 0\right)$, within the background field formulation, it is sufficient to evaluate covariant supergraphs for the case when $\Phi$ and $\bar{\Phi}$ are constant, while $W_{\alpha}$ and $\bar{W}_{\dot{\alpha}}$ obey the conditions (5). ${ }^{2}$ Indeed, the use of such a background allows one to keep track, in loop calculations, of the following sector

$$
\int \mathrm{d}^{8} z \frac{\bar{W}^{2} W^{2}}{\bar{\Phi}^{2} \Phi^{2}} \Omega\left(\Psi^{2}, 0\right)
$$

of the effective action. Once this functional form has been computed, one can remove the condition of relaxed super self-duality and work with arbitrary off-shell superfields. At the component level, the bosonic part of the functional is then

$$
\int \mathrm{d}^{4} x\left(F_{+}^{2} F_{-}^{2} / \Delta^{2}\right) \Omega\left(F_{+}^{2} / \Delta^{2}, 0\right), \quad \Delta=\bar{\varphi} \varphi,
$$

modulo terms involving the auxiliary fields and derivatives of $\varphi$ and $\bar{\varphi}$. For these reasons, relaxed super self-duality proves to be useful in Minkowski space.

In this paper, our attention will be restricted to the consideration of the real part of the effective action. At the one-loop level, the function $\Omega$ in $(8)$ is $[12,15]$ (see also $[16,17])$

$$
\Omega_{\text {one-loop }}\left(\Psi^{2}, \bar{\Psi}^{2}\right)=\frac{1}{(4 \pi)^{2}} \int_{0}^{\infty} \mathrm{d} s s \zeta(s \Psi, s \bar{\Psi}) \mathrm{e}^{-s},
$$

\footnotetext{
${ }^{2}$ In the case of $\mathcal{N}=4 S U(N)$ super Yang-Mills theory on its Coulomb branch, the choice of such a background tremendously simplifies the evaluation of the two-loop effective action [14].
} 
where

$$
\zeta(x, y)=\zeta(y, x)=\frac{y^{2}(\cosh x-1)-x^{2}(\cosh y-1)}{x^{2} y^{2}(\cosh x-\cosh y)} .
$$

From this,

$$
\Omega_{\text {one-loop }}\left(\Psi^{2}, 0\right)=\frac{1}{4(4 \pi)^{2}} \int_{0}^{\infty} \mathrm{d} s s\left\{\frac{1}{(s \Psi / 2)^{2}}-\frac{1}{\sinh ^{2}(s \Psi / 2)}\right\} \mathrm{e}^{-s} .
$$

In terms of the function [5]

$$
\xi(x)=-x\left(\frac{\mathrm{d}}{\mathrm{d} x} \ln \Gamma(x)-\ln x+\frac{1}{2 x}\right)=\frac{1}{2} \int_{0}^{\infty} \mathrm{d} s\left\{\frac{1}{s^{2}}-\frac{1}{\sinh ^{2} s}\right\} \mathrm{e}^{-2 x s},
$$

the $\Omega_{\text {one-loop }}\left(\Psi^{2}, 0\right)$ is seen to be proportional to the first derivative of $\xi$. What happens at two loops?

To answer this, we turn to the two-loop effective action for $\mathcal{N}=2$ SQED which was computed in [15] on the base of the covariant multi-loop technique of [18]:

$$
\begin{aligned}
\Gamma_{\text {two-loop }} & =\int \mathrm{d}^{8} z \frac{\bar{W}^{2} W^{2}}{\bar{\Phi}^{2} \Phi^{2}} \Omega_{\text {two-loop }}\left(\Psi^{2}, \bar{\Psi}^{2}\right), \\
\Omega_{\text {two-loop }}\left(\Psi^{2}, \bar{\Psi}^{2}\right) & =\Omega^{\mathrm{I}+\mathrm{II}}\left(\Psi^{2}, \bar{\Psi}^{2}\right)+\Omega^{\mathrm{III}}\left(\Psi^{2}, \bar{\Psi}^{2}\right) .
\end{aligned}
$$

Here $\Omega^{\mathrm{I}+\mathrm{II}}$ and $\Omega^{\mathrm{III}}$ correspond to different two-loop supergraphs [15], and have the form

$$
\begin{aligned}
\Omega^{\mathrm{I}+\mathrm{II}}\left(\Psi^{2}, \bar{\Psi}^{2}\right) & =\frac{e^{2}}{(4 \pi)^{4}} \int_{0}^{\infty} \mathrm{d} s \int_{0}^{\infty} \mathrm{d} t \mathrm{e}^{-(s+t)} \frac{s \lambda_{+}}{\sinh \left(s \lambda_{+}\right)} \frac{s \lambda_{-}}{\sinh \left(s \lambda_{-}\right)} \frac{t \lambda_{+}}{\sinh \left(t \lambda_{+}\right)} \frac{t \lambda_{-}}{\sinh \left(t \lambda_{-}\right)} \\
& \times \frac{\sinh (s \Psi / 2)}{s \Psi / 2} \frac{\sinh (s \bar{\Psi} / 2)}{s \bar{\Psi} / 2} \frac{\sinh (t \Psi / 2)}{t \Psi / 2} \frac{\sinh (t \bar{\Psi} / 2)}{t \bar{\Psi} / 2} I_{2}(s, t),
\end{aligned}
$$

and

$$
\begin{aligned}
\Omega^{\mathrm{III}}\left(\Psi^{2}, \bar{\Psi}^{2}\right) & =\frac{e^{2}}{2(4 \pi)^{4}} \int_{0}^{\infty} \mathrm{d} s \int_{0}^{\infty} \mathrm{d} t \mathrm{e}^{-(s+t)} \frac{s \lambda_{+}}{\sinh \left(s \lambda_{+}\right)} \frac{s \lambda_{-}}{\sinh \left(s \lambda_{-}\right)} \frac{t \lambda_{+}}{\sinh \left(t \lambda_{+}\right)} \frac{t \lambda_{-}}{\sinh \left(t \lambda_{-}\right)} \\
& \times\left\{\frac{\sinh ^{2}(s \Psi / 2)}{(s \Psi / 2)^{2}} \frac{\sinh ^{2}(t \bar{\Psi} / 2)}{(t \bar{\Psi} / 2)^{2}}+(s \leftrightarrow t)\right\} I_{1}(s, t),
\end{aligned}
$$

where

$$
\lambda_{ \pm}=\frac{1}{2}(\bar{\Psi} \pm \Psi),
$$

and $I_{1}(s, t)$ and $I_{2}(s, t)$ denote the following simple proper-time integrals

$$
\begin{aligned}
& I_{1}(s, t)=\int_{0}^{\infty} \frac{\mathrm{d} u}{u^{2}} \frac{1}{\left(u^{-1}+a_{+}\right)\left(u^{-1}+a_{-}\right)}, \\
& I_{2}(s, t)=\int_{0}^{\infty} \frac{\mathrm{d} u}{u^{2}} \frac{1}{\left(u^{-1}+a_{+}\right)\left(u^{-1}+a_{-}\right)}\left(\frac{P_{+}}{u^{-1}+a_{+}}+\frac{P_{-}}{u^{-1}+a_{-}}\right),
\end{aligned}
$$


with

$$
a_{ \pm}=\lambda_{ \pm} \operatorname{coth}\left(s \lambda_{ \pm}\right)+\lambda_{ \pm} \operatorname{coth}\left(t \lambda_{ \pm}\right), \quad P_{ \pm}=\frac{\lambda_{ \pm}}{\sinh \left(s \lambda_{ \pm}\right)} \frac{\lambda_{ \pm}}{\sinh \left(t \lambda_{ \pm}\right)}
$$

We are going to evaluate $\Omega_{\text {two-loop }}\left(\Psi^{2}, 0\right)$. Since

$$
\bar{\Psi}=0 \quad \longrightarrow \quad \lambda_{ \pm}= \pm \frac{1}{2} \Psi
$$

then

$$
a_{ \pm}=\frac{\Psi}{2} \frac{\sinh ((s+t) \Psi / 2)}{\sinh (s \Psi / 2) \sinh (t \Psi / 2)}, \quad P_{ \pm}=\frac{\Psi / 2}{\sinh (s \Psi / 2)} \frac{\Psi / 2}{\sinh (t \Psi / 2)},
$$

and therefore

$$
\begin{aligned}
& I_{1}(s, t)=\frac{2}{\Psi} \frac{\sinh (s \Psi / 2) \sinh (t \Psi / 2)}{\sinh ((s+t) \Psi / 2)} \\
& I_{2}(s, t)=\frac{\sinh (s \Psi / 2) \sinh (t \Psi / 2)}{\sinh ^{2}((s+t) \Psi / 2)}
\end{aligned}
$$

For $\Omega^{\mathrm{I}+\mathrm{II}}\left(\Psi^{2}, 0\right)$ we therefore get

$$
\Omega^{\mathrm{I}+\mathrm{II}}\left(\Psi^{2}, 0\right)=\frac{e^{2}}{(4 \pi)^{4}} \int_{0}^{\infty} \mathrm{d} s \int_{0}^{\infty} \mathrm{d} t s t \mathrm{e}^{-(s+t)} \frac{(\Psi / 2)^{2}}{\sinh ^{2}((s+t) \Psi / 2)} .
$$

The double proper-time integral here can be reduced to a single integral, by introducing new integration variables, $\alpha$ and $\tau$, defined as follows (see, e.g., [19])

$$
s+t=\tau, \quad s-t=\tau \alpha, \quad \tau \in[0, \infty), \quad \alpha \in[-1,1],
$$

such that

$$
\int_{0}^{\infty} \mathrm{d} s \int_{0}^{\infty} \mathrm{d} t L(s, t)=\frac{1}{2} \int_{0}^{\infty} \mathrm{d} \tau \int_{-1}^{+1} \mathrm{~d} \alpha \tau L(s(\alpha, \tau), t(\alpha, \tau)) .
$$

This leads to

$$
\begin{aligned}
\Omega^{\mathrm{I}+\mathrm{II}}\left(\Psi^{2}, 0\right) & =\frac{e^{2}}{6(4 \pi)^{4}} \int_{0}^{\infty} \mathrm{d} s s^{3} \frac{(\Psi / 2)^{2}}{\sinh ^{2}(s \Psi / 2)} \mathrm{e}^{-s} \\
& =\frac{e^{2}}{6(4 \pi)^{4}}+\frac{e^{2}}{6(4 \pi)^{4}} \int_{0}^{\infty} \mathrm{d} s s^{3}\left\{\frac{(\Psi / 2)^{2}}{\sinh ^{2}(s \Psi / 2)}-\frac{1}{s^{2}}\right\} \mathrm{e}^{-s} .
\end{aligned}
$$

For $\Omega^{\mathrm{III}}\left(\Psi^{2}, 0\right)$ we obtain

$$
\Omega^{\mathrm{III}}\left(\Psi^{2}, 0\right)=\frac{e^{2}}{(4 \pi)^{4}} \int_{0}^{\infty} \mathrm{d} s \int_{0}^{\infty} \mathrm{d} t s^{2} \frac{(\Psi / 2) \sinh (t \Psi / 2)}{\sinh (s \Psi / 2) \sinh ((s+t) \Psi / 2)} \mathrm{e}^{-(s+t)} .
$$


Using the identity

$$
\frac{\sinh t}{\sinh s \sinh (s+t)}=\operatorname{coth} s-\operatorname{coth}(s+t),
$$

we can rewrite $\Omega^{\mathrm{III}}\left(\Psi^{2}, 0\right)$ as follows:

$$
\begin{aligned}
\Omega^{\mathrm{III}}\left(\Psi^{2}, 0\right) & =\frac{e^{2}}{(4 \pi)^{4}} \int_{0}^{\infty} \mathrm{d} t \mathrm{e}^{-t} \int_{0}^{\infty} \mathrm{d} s s^{2}(\Psi / 2) \operatorname{coth}(s \Psi / 2) \mathrm{e}^{-s} \\
& -\frac{e^{2}}{(4 \pi)^{4}} \int_{0}^{\infty} \mathrm{d} s \int_{0}^{\infty} \mathrm{d} t s^{2}(\Psi / 2) \operatorname{coth}((s+t) \Psi / 2) \mathrm{e}^{-(s+t)} .
\end{aligned}
$$

In the first term here, one of the proper-time integrals is elementary. In the second term, the double proper-time integral can be reduced to a single one by implementing the change of variables (26). This gives

$$
\begin{aligned}
\Omega^{\mathrm{III}}\left(\Psi^{2}, 0\right) & =\frac{e^{2}}{3(4 \pi)^{4}} \int_{0}^{\infty} \mathrm{d} s\left(3 s^{2}-s^{3}\right)(\Psi / 2) \operatorname{coth}(s \Psi / 2) \mathrm{e}^{-s} \\
& =\frac{e^{2}}{3(4 \pi)^{4}}+\frac{e^{2}}{3(4 \pi)^{4}} \int_{0}^{\infty} \mathrm{d} s s^{3}\left\{\frac{(\Psi / 2)^{2}}{\sinh ^{2}(s \Psi / 2)}-\frac{1}{s^{2}}\right\} \mathrm{e}^{-s} .
\end{aligned}
$$

Combining the results (28) and (32), we finally obtain

$$
\begin{aligned}
\Omega_{\text {two-loop }}\left(\Psi^{2}, 0\right) & =\frac{e^{2}}{2(4 \pi)^{4}} \\
& +\frac{e^{2}}{2(4 \pi)^{4}} \int_{0}^{\infty} \mathrm{d} s s^{3}(\Psi / 2)^{2}\left\{\frac{1}{\sinh ^{2}(s \Psi / 2)}-\frac{1}{(s \Psi / 2)^{2}}\right\} \mathrm{e}^{-s} .
\end{aligned}
$$

This should be compared with the one-loop result (12). The second term in (33) is seen to be proportional to the third derivative of the function $(13)$, while $\Omega_{\text {one-loop }}\left(\Psi^{2}, 0\right)$ was proportional to the first derivative of the same function $\xi$. It is natural to wonder: Does this pattern persist at higher loops, so that the loop expansion for $\Omega\left(\Psi^{2}, 0\right)$ is equivalent to a derivative expansion of $\xi$ ?

The first term in (33) generates a non-vanishing $F^{4}$ quantum correction, while the other term produces $F^{6}$ and higher powers of the field strength. The two-loop $F^{4}$ term was also computed in [15] using the background field formulation in $\mathcal{N}=2$ harmonic superspace [20] in conjunction with the results of [21]. In spite of the expectations of [22], non-vanishing two-loop $F^{4}$ quantum corrections also appear in some $\mathcal{N}=2$ superconformal theories in four dimensions [23]. 


\section{Acknowledgements:}

We are grateful to the referee for constructive comments and for pointing out misprints. This work is supported in part by the Australian Research Council and UWA research grants.

\section{References}

[1] W. Heisenberg and H. Euler, "Folgerungen aus der Diracschen Theorie des Positrons," Z. Phys. 98 (1936) 714.

[2] V. Weisskopf, "Über die Elektrodynamik des Vakuums auf Grund der Quantentheorie des Elektrons," Kong. Dans. Vid. Selsk. Math-fys. Medd. XIV No. 6 (1936), reprinted in Quantum Electrodynamics, J. Schwinger (Ed.), Dover, New York, 1958.

[3] J. S. Schwinger, "On gauge invariance and vacuum polarization," Phys. Rev. 82 (1951) 664.

[4] V. I. Ritus, "Lagrangian of an intense electromagnetic field and quantum electrodynamics at short distances," Sov. Phys. JETP 42 (1975) 774.

[5] G. V. Dunne and C. Schubert, "Closed-form two-loop Euler-Heisenberg Lagrangian in a self-dual background," Phys. Lett. B 526 (2002) 55 [arXiv:hep-th/0111134]; "Two-loop self-dual Euler-Heisenberg Lagrangians. I: Real part and helicity amplitudes," JHEP 0208 (2002) 053 [arXiv:hep-th/0205004]; "Two-loop self-dual EulerHeisenberg Lagrangians. II: Imaginary part and Borel analysis," JHEP 0206 (2002) 042 [arXiv:hep-th/0205005]; G. V. Dunne, H. Gies and C. Schubert, "Zero modes, beta functions and IR/UV interplay in higher-loop QED," JHEP 0211 (2002) 032 [arXiv:hep-th/0210240]; G. V. Dunne, "Two-loop diagrammatics in a self-dual background," arXiv:hep-th/0311167.

[6] M. T. Grisaru, H. N. Pendleton and P. van Nieuwenhuizen, "Supergravity and the S matrix," Phys. Rev. D 15 (1977) 996; M. T. Grisaru and H. N. Pendleton, "Some properties of scattering amplitudes in supersymmetric theories," Nucl. Phys. B 124 (1977) 81.

[7] S. M. Christensen, S. Deser, M. J. Duff and M. T. Grisaru, "Chirality, self-duality, and supergravity counterterms," Phys. Lett. B 84 (1979) 411. 
[8] M. J. Duff and C. J. Isham, "Self-duality, helicity, and coherent states in non-Abelian gauge theories," Nucl. Phys. B 162 (1980) 271; "Self-duality, helicity, and supersymmetry: The scattering of light by light," Phys. Lett. B 86 (1979) 157.

[9] R. E. Kallosh, "Super-self-duality," JETP Lett. 29 (1979) 172; "Self-duality in superspace," Nucl. Phys. B 165 (1980) 119.

[10] B. Zumino, "Euclidean supersymmetry and the many-instanton problem," Phys. Lett. B 69 (1977) 369.

[11] W. Siegel, Fields, arXiv:hep-th/9912205.

[12] I. L. Buchbinder, S. M. Kuzenko and A. A. Tseytlin, "On low-energy effective actions in $\mathrm{N}=2,4$ superconformal theories in four dimensions," Phys. Rev. D 62 (2000) 045001 [arXiv:hep-th/9911221].

[13] I. L. Buchbinder, S. M. Kuzenko and B. A. Ovrut, "On the D = 4, N=2 nonrenormalization theorem," Phys. Lett. B 433 (1998) 335 [arXiv:hep-th/9710142].

[14] S. M. Kuzenko and I. N. McArthur, "Relaxed super self-duality and N=4 SYM at two loops," arXiv:hep-th/0403240.

[15] S. M. Kuzenko and I. N. McArthur, "Low-energy dynamics in N = 2 super QED: Two-loop approximation," JHEP 0310 (2003) 029 [arXiv:hep-th/0308136].

[16] I. N. McArthur and T. D. Gargett, "A 'Gaussian' approach to computing supersymmetric effective actions," Nucl. Phys. B 497 (1997) 525 [arXiv:hep-th/9705200].

[17] N. G. Pletnev and A. T. Banin, "Covariant technique of derivative expansion of the one-loop effective action," Phys. Rev. D 60 (1999) 105017 [arXiv:hep-th/9811031].

[18] S. M. Kuzenko and I. N. McArthur, "On the background field method beyond one loop: A manifestly covariant derivative expansion in super Yang-Mills theories," JHEP 0305 (2003) 015 [arXiv:hep-th/0302205].

[19] W. Dittrich and M. Reuter, Effective Lagrangians in Quantum Electrodynamics, Springer, Berlin, 1985.

[20] I. L. Buchbinder, E. I. Buchbinder, S. M. Kuzenko and B. A. Ovrut, "The background field method for $\mathrm{N}=2$ super Yang-Mills theories in harmonic superspace," Phys. Lett. B 417 (1998) 61 [arXiv:hep-th/9704214]. 
[21] S. M. Kuzenko and I. N. McArthur, "Effective action of N $=4$ super Yang-Mills: N = 2 superspace approach," Phys. Lett. B 506 (2001) 140 [arXiv:hep-th/0101127]; "Hypermultiplet effective action: $\mathrm{N}=2$ superspace approach," Phys. Lett. B 513 (2001) 213 [arXiv:hep-th/0105121].

[22] M. Dine and N. Seiberg, "Comments on higher derivative operators in some SUSY field theories," Phys. Lett. B 409 (1997) 239 [arXiv:hep-th/9705057].

[23] S. M. Kuzenko and I. N. McArthur, "On the two-loop four-derivative quantum corrections in 4D N = 2 superconformal field theories," Nucl. Phys. B 683 (2004) 3 [arXiv:hep-th/0310025]. 